\title{
Two interesting species of the genus Harpactea from Crimea (Aranei: Dysderidae)
}

\author{
Ава интересных вида рода Harpactea из Крыма \\ (Aranei: Dysderidae)
}

\author{
Mykola M. Kovblyuk, Zoya A. Kastrygina \\ Н.М. Ковблюк, 3.А. Кастрыгина
}

Zoology Department, V.I. Vernadsky Taurida National University, Yaltinskaya Street 4, Simferopol 95007, Ukraine. E-mail:
kovblyuk@mail.ru; zoiac_21@mail.ru
Кафедра зоологии, Таврический национальный университет им. В.И. Вернадского, ул. Ялтинская 4, Симферополь 95007, Украина.

KEY WORDS: spiders, Harpactea, new records, redescriptions, Crimea.

КЛЮЧЕВЫЕ СЛОВА: пауки, Harpactea, новые находки, переописания, Крым.

ABSTRACT. Harpactea alexandrae Lazarov, 2006 and H. longobarda Pesarini, 2001 are found in the Opuk Nature Reserve in Crimea, which represents the easternmost limit of their distribution. Both are recorded from Crimea, Ukraine and the former Soviet Union for the first time. The illustrated redescriptions of both species are provided. An updated checklist of the Crimean dysderids is presented.

РЕЗЮМЕ. Harpactea alexandrae Lazarov, 2006 и H. longobarda Pesarini, 2001 обнаружены в Опукском заповеднике в Крыму, который является самой восточной точкой их распространения. Они впервые отмечены для Крыма, Украины и бывшего Советского Союза. Для обоих видов даны иллюстрированные переописания. Представлен обновлённый список дисдерид Крыма.

\section{Introduction}

This paper is a continuation of our studies of the Crimean dysderid spiders [Kovblyuk, 2002; Kovblyuk \& Nadolny, 2007; Nadolny \& Kovblyuk, 2007; Kovblyuk et al., 2008]. So far, nine dysderid species belonging to two genera (Dysdera Latreille, 1804 and Harpactea Bristowe, 1939) have been reported from Crimea. Two additional, poorly-known species were found in the Opuk Nature Reserve, and both are redescribed in this paper.

\section{Material and methods}

The specimens treated in this study are housed in the collections of Zoology Department, V.I. Vernadsky Taurida National University, Simferopol, Ukraine, curator M.M. Kovblyuk (TNU) and the Zoological Museum of the Moscow State University, Moscow, Russia, curator K.G. Mikhailov (ZMMU). In the material reported below the name of the collector V.A. Gnelitsa is abbreviated as V.G.

Leg segments were measured after their separation from the cephalothorax. All measurements are in $\mathrm{mm}$ : minimum-maximum; figures in brackets represent mean values.

Drawings were made by means of both dissecting and compound microscopes using a grid method. All scale bars are $0.1 \mathrm{~mm}$. The illustrations of endogyne were made after their maceration in the $20 \%$ water solution of $\mathrm{KOH}$.

The morphological terminology and abbreviations follows Dunin [1992]: $A D$ - anterior diverticulum; $C$ conductor; $C h$ - ventral medial endochondrite; $E$ embolus; $P D$ - posterior diverticulum; $P S p$ - paraspermatheca; $S p$ - spermatheca; $T$ - terminal apophysis of conductor. Positions of leg spines: $d$ - dorsal; $\mathrm{p}$ - prolateral; $\mathrm{r}$ - retrolateral; v — ventral.

\section{Species survey}

Harpactea Bristowe, 1939

Harpactea alexandrae Lazarov, 2006

Figs 1-3, 8 .

H. a. Lazarov, 2006: 13, figs 2-4 $\left(\sigma^{7}\right)$. ( $\left.\sigma^{\top}+\right)$.

H. a.: Spiegelaere \& Bosmans, 2009: 8, figs 1A-B, 2, 3A-C

H. a.: Le Peru, 2011: 192, 263, fig. $378\left(0^{7}\right)$.

MATERIAL. UKRAINE. Crimea, Lenino District, Kerch Peninsula, Opuk Nature Reserve: $1 \sigma^{7}, 4$ क् (TNU-3091/5), stones, Rosa, 24.04.2005, V.G.; 1 क (TNU-3088/4), same locality, 24.04.2005, V.G.; 2 우 (ZMMU from TNU-3083/3), N slope of Opuk Mt., 27.04.2005, V.G.

DIAGNOSIS. Similar to Harpactea doblikae (Thorell, 1875) and H. rubicunda (C.L. Koch, 1838) [Kovblyuk et al., 2008: figs 88-91 and 107-110; Le Peru, 2011: figs 399 and 437], but differs in the shape of copulatory organs (Figs 1-3). 


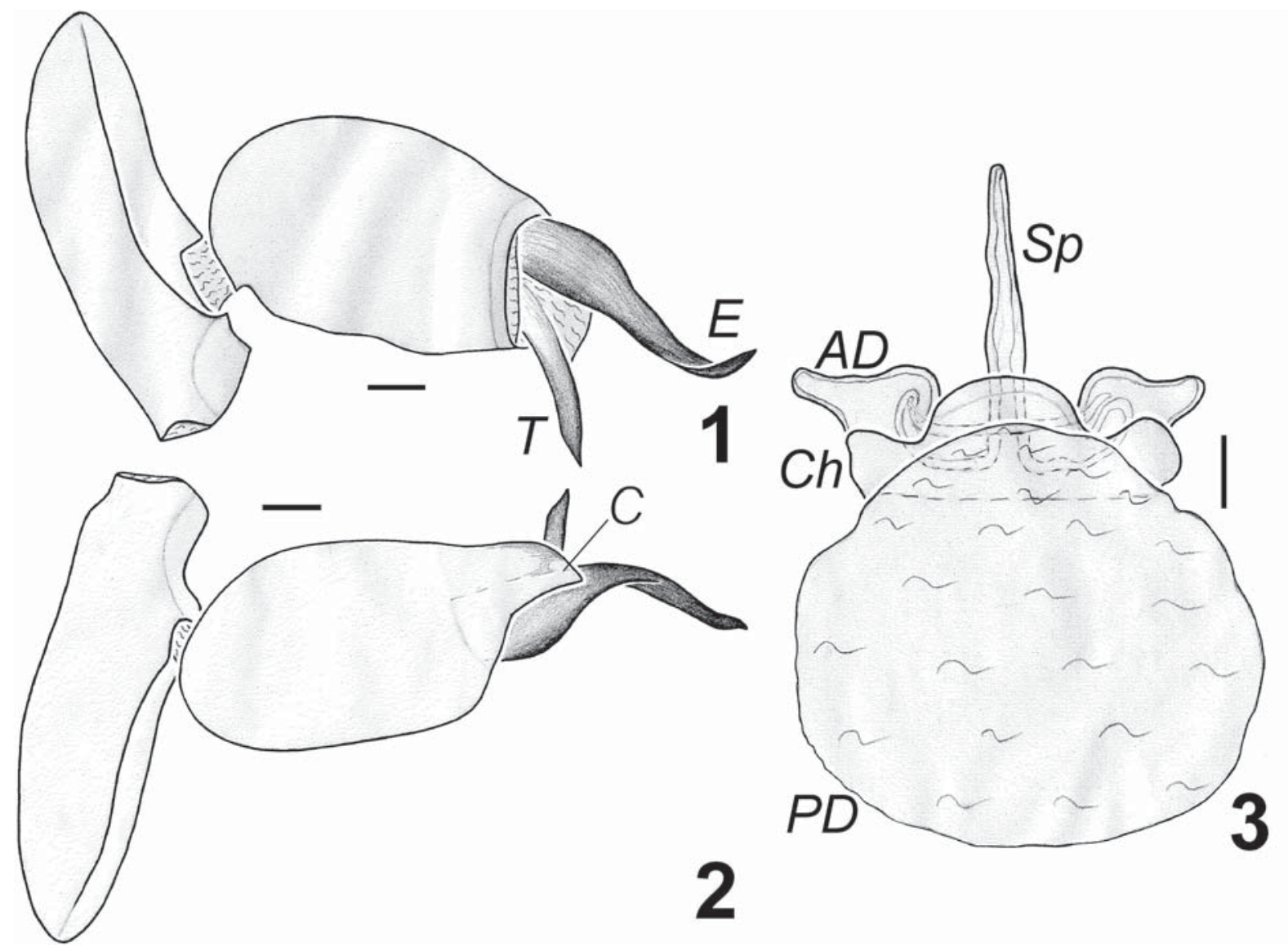

Figs 1-3. Copulatory organs of Harpactea alexandrae: 1 - palp, retrolateral view; 2 - palp, prolateral view; 3 - endogyne, dorsal view. Scale $0.1 \mathrm{~mm}$. Abbreviations: $A D$ - anterior diverticulum; $C$ - conductor; $C h$ - ventral medial endochondrite; $E-$ embolus; $P D$ - posterior diverticulum; $S p$ - spermatheca; $T$ - terminal apophysis of conductor.

Рис. 1-3. Копулятивные органы Harpactea alexandrae: 1 - пальпа, ретролатерально; 2 - пальпа, пролатерально; 3 эндогина, дорсально. Масштаб 0,1 мм. Обозначения: $A D-$ передний дивертикул; $C$ - кондуктор; Ch- вентральный медиальный эндохондрит; $E$ - эмболюс; $P D$ - задний дивертикул; $S p$ - сперматека; $T$ - терминальный апофиз кондуктора.

DESCRIPTION. Male $(\mathrm{n}=1)$ and females $(\mathrm{n}=2)$ measurements $\left(O^{7} /\right.$ / $)$ : total length 5.9 / 6.2-10.5 (8.4); carapace 3.0 / 2.8-3.4 (3.1) long, 2.3 / 2.2-2.8 (2.5) wide. Chelicerae length $1.4 / 1.2-1.5$ (1.3). Lengths of female leg segments as in Table 1.

Chelicerae with 2 promarginal and 2 retromarginal teeth in both sexes. Male leg spination as in Table 2.

Female leg spination as in Table 3.

DISTRIBUTION. This species is known from the Black Sea coast of Romania (Constanca), north-eastern Bulgaria [Lazarov, 2006; Spiegelaere \& Bosmans, 2009] and Crimea [present data] (Fig. 8).

COMMENTS. This species is recorded from Crimea, Ukraine and the former Soviet Union for the first time. Crimea is the easternmost locality of the $H$. alexandrae distribution (Fig. 8).

HABITATS. In Crimea, $H$. alexandrae was found in the steppe with Rosa bushes. In Bulgaria this species was also found in dry stony areas with bushes [Lazarov, 2006], contrary to Romania where it was record- ed from the dune grasslands [Spiegelaere \& Bosmans, 2009].

PHENOLOGY. In Crimea: $\bigcirc^{7}+$ - IV. In Romania: $\sigma^{\top} 9$ - V-VI [Spiegelaere \& Bosmans, 2009], one month later than in Crimea. In Bulgaria: $\sigma^{7} \sigma^{7}-\mathrm{VI}-$ VII, XI [Lazarov, 2006], two months later than in Crimea.

Harpactea longobarda Pesarini, 2001

Figs 4-7, 8.

H. l. Pesarini, 2001: 300, figs 18-19 ( (

H. l.: Le Peru, 2011: 199, 274, figs 423 ( $\left.\sigma^{7}+\right)$.

MATERIAL. UKRAINE. Crimea, Lenino District, Kerch Peninsula, Opuk Nature Reserve: $1 \sigma^{7}, 1$ (ZMMU from TNU3079/2), S slope Opuk Mt., near the stream, 18.04.2005, V.G.; 1 ऽ , 2 우 (TNU-3082/8), S slope Opuk Mt., stones, 19.04.2005, V.G; $2 \sigma^{7}, 1$ (TNU-3104/2), same reserve, 20.04.2005, V.G.; $2 \sigma^{\top} \sigma^{\top}, 1$ ㅇ (TNU-3072/1), steppe, 21.04.2005, V.G.; 1 ㅇ (TNU-3076/4), Opuk Mt., Celtis glabrata, Rosa, Prunus stepposa, under moss, 22.04.2005, V.G; 2 O$^{7} \sigma^{7}, 3$ 우 (TNU-3096/ 4), Opuk Mt., ravine, meadow, 23.04.2005, V.G.; $1 \sigma^{\top}, 1$ ㅇ 
Table 1. Lengths of female leg segments of Harpactea alexandrae.

Таблица 1. Длина члеников ног самок Harpactea alexandrae.

\begin{tabular}{|l|c|c|c|c|c|c|}
\hline & femur & patella & tibia & metatarsus & tarsus & Total \\
\hline I & $2.5-3.0(2.7)$ & $1.6-1.8(1.7)$ & $2.1-2.5(2.3)$ & $2.0-2.4(2.2)$ & 0.6 & $8.8-10.4(9.6)$ \\
\hline II & $2.3-2.6(2.4)$ & $1.4-1.7(1.6)$ & $2.0-2.3(2.1)$ & $1.9-2.4(2.1)$ & $0.5-0.6(0.6)$ & $8.1-9.6(8.8)$ \\
\hline III & $1.9-2.1(2.0)$ & $1.0-1.2(1.1)$ & $1.4-1.5(1.4)$ & $1.9-2.0(2.0)$ & $0.5-0.6(0.6)$ & $6.7-7.4(7.0)$ \\
\hline IV & $2.7-3.0(2.8)$ & $1.3-1.6(1.4)$ & $2.3-2.4(2.4)$ & $2.8-2.9(2.8)$ & $0.6-0.7(0.7)$ & $9.7-10.6(10.1)$ \\
\hline
\end{tabular}

Table 2. Male leg spination of Harpactea alexandrae. Таблица 2. Вооружение самца Harpactea alexandrae.

\begin{tabular}{|c|c|c|c|c|c|}
\hline & femur & patella & tibia & metatarsus & tarsus \\
\hline Leg I & p 1-2 & 0 & 0 & 0 & 0 \\
\hline Leg II & p 1-1-2 & 0 & 0 & 0 & 0 \\
\hline Leg III & $\begin{array}{l}\text { d } 1 ; \\
\text { p } 1-1-1 ; \\
\text { r } 1-1-1-1\end{array}$ & p 1 & $\begin{array}{l}\text { p } 2-1-1-1 ; \\
\text { r 2-1-1-1; } \\
\text { v } 2-2-2\end{array}$ & $\begin{array}{l}\text { p } 1-1-1 ; \\
\text { r } 1-1 ; \\
\text { v } 1-1-1-1-2\end{array}$ & 0 \\
\hline Leg IV & $\begin{array}{l}\text { d } 1-1-2-1 ; \\
\text { p } 1-1 ; \\
\text { r } 1-1-1-1\end{array}$ & $\mathrm{p} 1$ & $\begin{array}{l}\text { p } 2-1-2 ; \\
\text { r 2-2-1-1-1; } \\
\text { v 3-1-1-2-2 }\end{array}$ & $\begin{array}{l}\text { p } 1-1-1-1 ; \\
\text { r } 1-1-1 ; \\
\text { v } 1-2-1-1-2-2\end{array}$ & 0 \\
\hline
\end{tabular}

Table 3. Female leg spination of Harpactea alexandrae. Таблица 3. Вооружение ног самок Harpactea alexandrae.

\begin{tabular}{|c|c|c|c|c|c|}
\hline & femur & patella & tibia & metatarsus & tarsus \\
\hline Leg I & p 2 or $2-2$ & 0 & 0 & 0 & 0 \\
\hline Leg II & p $1-2$ or $1-1-2$ & 0 & 0 & 0 & 0 \\
\hline Leg III & $\begin{array}{l}\text { d } 1 \text { or } 0 ; \\
\text { p } 1-1 \text { or } 1-1-1 ; \\
\text { r } 0 \text { or } 1-1\end{array}$ & $\mathrm{p} 1$ & $\begin{array}{l}\text { p } 1-1-2 \text { or } 1-1-1-1 ; \\
\text { r } 1-1 \text { or } 1-1-1 ; \\
\text { v } 1-1-2 \text { or } 2-1-1-2\end{array}$ & $\begin{array}{l}\text { p } 1-1-1 ; \\
\text { r } 1-1 \text { or } 1-1-1 \text {; } \\
\text { v } 2-2 \text { or } 2-1-2\end{array}$ & 0 \\
\hline Leg IV & $\begin{array}{l}\text { d } 1 \text { or } 1-1-1 ; \\
\text { p } 0 \text { or } 1-1 ; \\
\text { r } 1-1-1 \text { or } 1-1-1-1\end{array}$ & $\begin{array}{l}\mathrm{p} 0 \text { or } \\
1\end{array}$ & $\begin{array}{l}\text { p } 1-1 \text { or } 1-1-1 ; \\
\text { r } 1-1-1 \text { or } 2-2-2 ; \\
\text { v } 1-1-2 \text { or } 3-1-1-2-2\end{array}$ & $\begin{array}{l}\text { p } 1-1-1-1 ; \\
\text { r } 1-1-1 \text { or } 1-2-1-1 \text {; } \\
\text { v } 1-1-1-2 \text { or } 1-2-2\end{array}$ & 0 \\
\hline
\end{tabular}

Table 4. Length of leg segments $\left(\mathrm{O}^{\top} / \mathrm{O}\right)$ of Harpactea longobarda.

Таблица 4. Длина члеников ног (О / + ) Harpactea longobarda.

\begin{tabular}{|l|l|l|l|l|l|l|}
\hline & \multicolumn{1}{|c|}{ femur } & \multicolumn{1}{c|}{ patella } & \multicolumn{1}{c|}{ tibia } & \multicolumn{1}{c|}{ metatarsus } & \multicolumn{1}{c|}{ tarsus } & \multicolumn{1}{c|}{ Total } \\
\hline \multirow{2}{*}{ I } & $1.5-1.9(1.7) /$ & $0.9-1.2(1.0) /$ & $1.3-1.6(1.4) /$ & $1.2-1.6(1.4) /$ & $0.4-0.5(0.5) /$ & $5.4-6.8(6.1) /$ \\
& $1.6-1.8(1.7)$ & $1.0-1.2(1.1)$ & $1.3-1.5(1.4)$ & $1.2-1.5(1.4)$ & $0.4(0.4)$ & $5.5-6.5(6.0)$ \\
\hline \multirow{2}{*}{ II } & $1.4-1.8(1.6) /$ & $0.8-1.1(0.9) /$ & $1.2-1.5(2.0) /$ & $1.2-1.6(1.4) /$ & $0.4-0.5(0.4) /$ & $4.9-6.4(5.6) /$ \\
& $1.5-1.7(1.6)$ & $0.9-1.1(1.0)$ & $1.2-1.4(1.3)$ & $1.2-1.5(1.3)$ & $0.4-0.4(0.4)$ & $5.3-6.2(5.8)$ \\
\hline \multirow{2}{*}{ III } & $1.1-1.4(1.2) /$ & $0.6-0.7(0.6) /$ & $0.8-1.0(0.9) /$ & $1.1-1.4(1.3) /$ & $0.4(0.4) /$ & $3.9-4.9(4.4) /$ \\
& $1.2-1.4(1.3)$ & $0.6-0.8(0.7)$ & $0.9-1.0(1.0)$ & $1.2-1.4(1.3)$ & $0.4(0.4)$ & $4.3-5.1(4.7)$ \\
\hline \multirow{2}{*}{ IV } & $1.5-1.9(1.7) /$ & $0.7-0.9(0.8) /$ & $1.3-1.6(1.4) /$ & $1.5-2.0(1.7) /$ & $0.5(0.5) /$ & $5.5-6.9(6.2) /$ \\
& $1.7-2.0(1.9)$ & $0.8-1.0(0.9)$ & $1.5-1.7(1.6)$ & $1.8-2.0(1.9)$ & $0.5(0.5)$ & $6.3-7.3(6.8)$ \\
\hline
\end{tabular}

Table 5. Male leg spination of Harpactea longobarda. Таблица 5. Вооружение ног самцов Harpactea longobarda.

\begin{tabular}{|c|c|c|c|c|c|}
\hline & femur & patella & tibia & metatarsus & tarsus \\
\hline Leg I & p 1-2 & 0 & 0 & 0 & 0 \\
\hline Leg II & p 1-1 or $1-1-1$ & 0 & 0 & 0 & 0 \\
\hline Leg III & $\begin{array}{l}\text { d } 0 \text { or } 1 \text { or } 1-1-1 ; \\
\text { p } 1-1-1 ; \\
\text { r } 1-1 \text { or } 1-1-1-1\end{array}$ & $\mathrm{p} 1$ & $\begin{array}{l}\text { d } 1 ; \\
\text { p } 1-1-1 ; \\
\text { r 1-1; } \\
\text { v 1-1-2 }\end{array}$ & $\begin{array}{l}\text { p 1-1-1; } \\
\text { r 1-1; } \\
\text { v 1-1-2 }\end{array}$ & 0 \\
\hline Leg IV & $\begin{array}{l}\text { d } 1-2-1 \text { or } 2-1- \\
1-1\end{array}$ & 0 & $\begin{array}{l}\text { d } 1 \text { or } 1-1 \text { or } 2-1-1 ; \\
\text { p } 1-1-1 ; \\
\text { r } 1-1-1 ; \\
\text { v } 2-1-2\end{array}$ & $\begin{array}{l}\text { p 1-1-1-1; } \\
\text { r 1-1-1; } \\
\text { v } 1-1-1-2\end{array}$ & 0 \\
\hline
\end{tabular}


Table 6. Female leg spination of Harpactea longobarda. Таблица 6. Вооружение ног самок Harpactea longobarda.

\begin{tabular}{|c|c|c|c|c|c|}
\hline & femur & patella & tibia & metatarsus & tarsus \\
\hline Leg I & $\mathrm{p} 1-1-1$ or $1-2$ & 0 & 0 & 0 & 0 \\
\hline Leg II & $\mathrm{p} 1-1-1$ & 0 & 0 & 0 & 0 \\
\hline Leg III & $\begin{array}{l}\text { d } 0 \text { or } 1 ; \\
\text { p } 1-1 \text { or } 1-1-1 \text {; } \\
\text { r } 1-1 \text { or } 1-1-1\end{array}$ & $\mathrm{p} 1$ & $\begin{array}{l}\text { d } 1 ; \\
\text { p } 1-1-1-1 ; \\
\text { r } 1-1 ; \\
\text { v } 1-1-2\end{array}$ & $\begin{array}{l}\text { p } 1-1-1 ; \\
\text { r } 1-1 ; \\
\text { v } 1-1-2 \text { or } 1-1-1-2\end{array}$ & 0 \\
\hline Leg IV & d $1-1-1-1-1-1$ or $1-2-1-1$ & 0 & $\begin{array}{l}\text { d } 1-1 ; \\
\text { p } 1-1-1 \text { or } 2-1-1 ; \\
\text { r } 1-1-1 ; \\
\text { v } 1-1-2 \text { or } 2-1-1-2\end{array}$ & $\begin{array}{l}\text { p } 1-1-1-1 ; \\
\text { r } 1-1-1 ; \\
\text { v } 1-1-1-2\end{array}$ & 0 \\
\hline
\end{tabular}

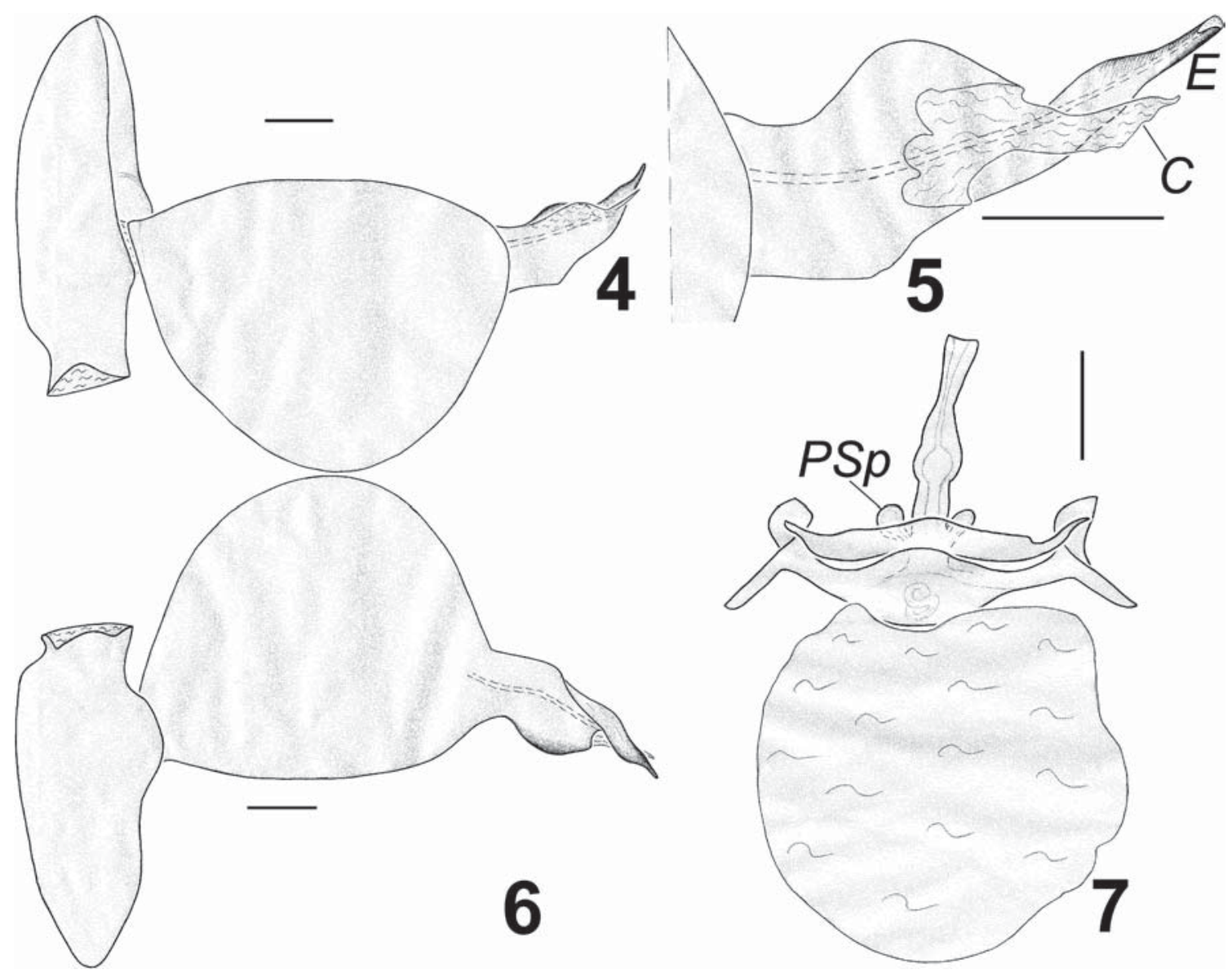

Figs 4-7. Copulatory organs of Harpactea longobarda: 4 - palp, prolateral view; 5 - embolar division, prolateral view; 6 - palp, retrolateral view; 7 - endogyne, dorsal view. Scale $0.1 \mathrm{~mm}$. Abbreviations: $C$ - conductor; $E$ - embolus; $P S p$ - paraspermatheca.

Рис. 4-7. Копулятивные органы Harpactea longobarda: 4 - пальпа, пролатерально; 5 - эмболярный отдел, пролатерально; 6 пальпа, ретролатерально; 7 - эндогина, дорсально. Масштаб 0,1 мм. Обозначения: $C$ - кондуктор; $E-$ эмболюс; $P S p$ парасперматека.

(ZMMU from TNU-3095/1), Opuk Mt., steppe, 23.04.2005, V.G.; $2 \sigma^{\top} \sigma^{\top}, 1$ क (TNU-3103/2), meadow, 23.04.2005, V.G.

DIAGNOSIS. This species is similar to H. aeoliensis Alicata, 1973 [Le Peru, 2011: fig. 374], but differs in the shape of copulatory organs (Figs 4-7).
DESCRIPTION. Males $(\mathrm{n}=3)$ and females $(\mathrm{n}=2)$ measurements $\left(O^{\top} /\right.$ O ): total length 3.5-4.4 (3.9) / 4.65.8 (5.2); carapace 1.7-2.2 (2.0) / 2.0-2.4 (2.2) long, 1.4-1.8 (1.6) / 1.6-1.8 (1.7) wide. Chelicerae length $0.7-0.9(0.8) / 0.8-1.0(0.9)$. 


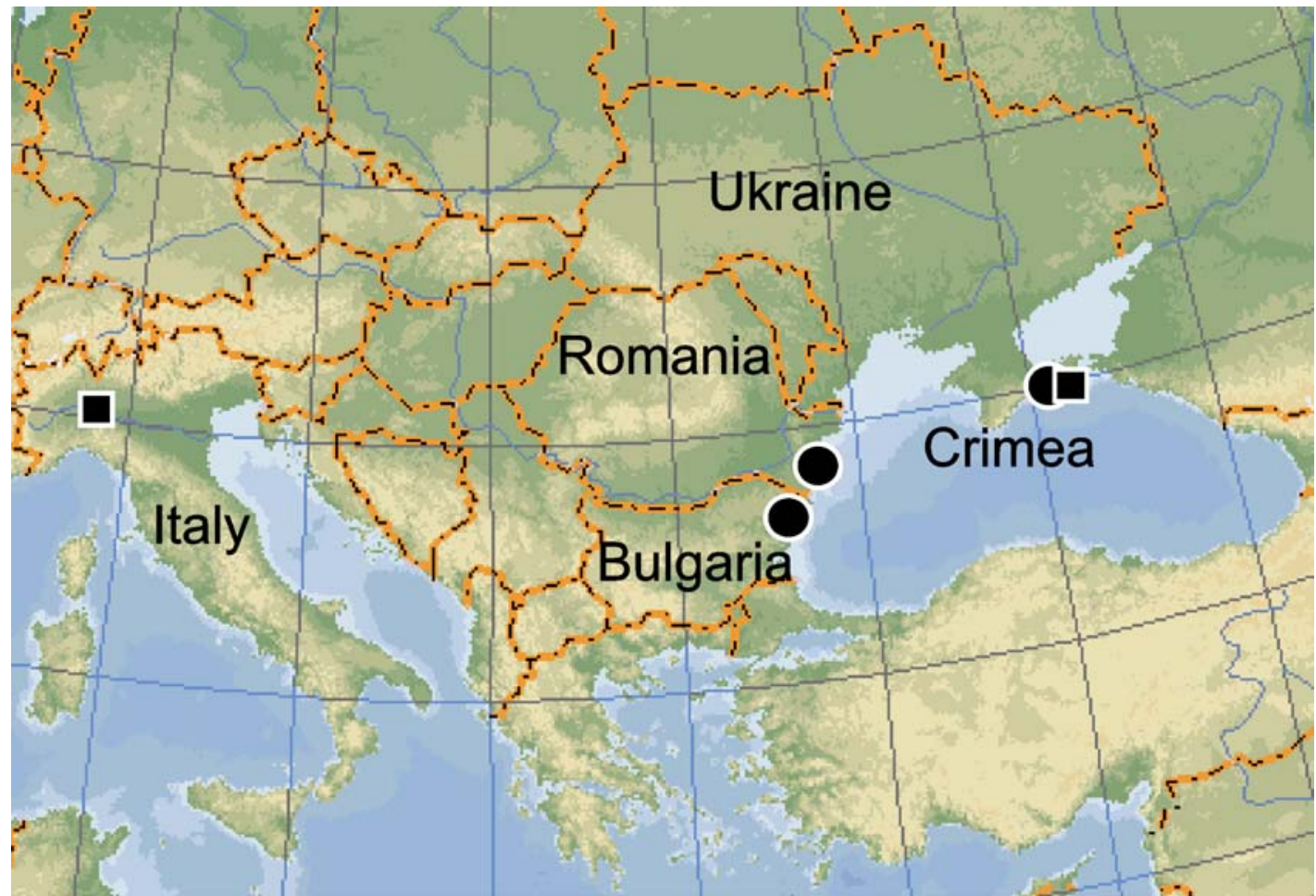

- H. alexsandrae

- H. longobarda

Fig. 8. Distribution of Harpactea alexandrae (circles) and H. longobarda (squares) based on literature and examined material. Some symbols indicate more then one locality.

Рис. 8. Распространение Harpactea alexandrae (кружки) и H. longobarda (квадраты) по литературным и собственным данным. Некоторые из символов соответствуют более чем одному локалитету.

Length of leg segments $\left(\sigma^{\top} /+\right)$ as in Table 4.

Chelicerae with 2 promarginal and 2 retromarginal teeth in both sexes.

Male leg spination as in Table 5.

Female leg spination as in Table 6.

DISTRIBUTION. This species is known from Italy (Lombardia) [Pesarini, 2001; Le Peru, 2011] and Crimea [present data] (Fig. 8).

COMMENTS. This species is recorded from Crimea, Ukraine and the former Soviet Union for the first time. Crimea is the easternmost locality of the H. longobarda distribution (Fig. 8).

HABITATS. In Crimea, this species was found in bushes, meadows and steppes.

PHENOLOGY. In Crimea: $\sigma^{\top} O+$ - IV. In Italy: $\sigma^{7}+$ - V, $\bigcirc^{\top} \bigcirc^{7}-$ III, X [Pesarini, 2001].

\section{Discussion}

\section{Checklist of the Crimean Dysderidae}

By now, 11 dysderid species have been recorded from Crimea [Kovblyuk, 2002; Kovblyuk \& Nadolny, 2007; Nadolny \& Kovblyuk, 2007; Kovblyuk et al., 2008; present data]. An updated checklist of the Dysderidae of Crimea is as follows:
Dysdera Latreille, 1804

1. D. crocata C.L. Koch, 1838;

2. D. dunini Deeleman-Reinhold, 1988;

3. D. hungarica Kulczyński in Chyzer et Kulczyński, 1897;

4. D. lata Wider in Reuss, 1834;

5. D. longirostris Doblika, 1853.

Harpactea Bristowe, 1939

6. H. alexandrae Lazarov, 2006;

7. H. azowensis Charitonov, 1956;

8. H. doblikae (Thorell, 1875);

9. H. longobarda Pesarini, 2001;

10. H. rubicunda (C.L. Koch, 1838);

11. H. spasskyi Dunin, 1992.

The distribution data and identification drawings for all the aforementioned species can be found in Kovblyuk et al. [2008] and the present paper.

\section{Zoogeography}

$H$. alexandrae is the first dysderid species that seems to display a Crimean-Balkan disjunctive range. To date, only four spider species having the similar CrimeanBalkan disjunctive ranges have been known: Amaurobius strandi Charitonov, 1937 (Amaurobiidae), Araeoncus tauricus Gnelitsa, 2004 (Linyphiidae), Zelotes 
eugenei Kovblyuk, 2009 (Gnaphosidae) and Zoropsis lutea (Thorell, 1875) (Zoropsidae).

$H$. longobarda has an unusual Crimean-Apennine disjunctive range. Such a distribution pattern has never been reported for a spider species to date. Actually, the species may have an Eastern Mediterranean range, however, it has not been found between Italy and Crimea yet.

ACKNOWLEDGEMENTS. We are very grateful to V.A. Gnelitsa (Ukraine, Sumy), who provided us with the material used in this study. We thank P.E. Gol'din (Simferopol) for improving the English of the earlier draft. Special thanks go to D.V. Logunov (Manchester, UK) who critically commented on the ms and edited its final version. This work was supported in part by the Karadag Nature Reserve.

\section{References}

Dunin P.M. 1992. The spider family Dysderidae of the Caucasian fauna (Arachnida Aranei Haplogynae) // Arthropoda Selecta. Vol.1. No.3. P.35-76.

Kovblyuk M.M. 2002. [Harpactea doblikae and H. rubicunda (Aranei, Dysderidae) in Crimea] // Vestnik zoologii. T.36. No.3. P.81-86 [in Russian, with English summary].

Kovblyuk M.M., Nadolny A.A. 2007. [Harpactea spasskyi (Aranei, Dysderidae) from the Crimea]// Vestnik zoologii. T.41. No.6. P.547-548 [in Russian, with English summary].
Kovblyuk M.M., Prokopenko E.V., Nadolny A.A. 2008. [Spider family Dysderidae of the Ukraine (Arachnida, Aranei)] // Euroasian entomological journal. Vol.7. No.4. P.287-306 [in Russian, with English summary].

Lazarov S. 2006. A new spiders from Bulgaria Harpactea alexandrae sp. n. (Araneae: Dysderidae) // Acta zoological bulgarica. Vol.58. Issue 1. P.13-16.

Le Peru B. 2011. The spiders of Europe, a synthesis of data: Volume 1 Atypidae to Theridiidae // Mémoires de la Société linnéenne de Lyon $\mathrm{n}^{\circ} 2.522$ pp.

Nadolny A.A., Kovblyuk M.M. 2007. [The landscape distribution and seasonal dynamic of activity of spider family Dysderidae (Aranei) in the Crimea] // Problemy i perspektivy obschei entomologii. Tezisy dokladov XIII s'ezda Russkogo entomologicheskogo obschestva, Krasnodar, 9-15 sentyabrya 2007. Krasnodar: Kubanskyi gosudarstvennyi agrarnyi universitet. P.242 [in Russian].

Platnick N.I. 2013. The world spider catalog, version 14.0. American Museum of Natural History; online at: http://research.amnh. org/entomology/spiders/catalog/index.html (accessed June 11, 2013).

Pesarini C. 2001. Sei nuove specie di Dysderidae d'Italia e di Grecia (Araneae) // Atti della Societa Italiana di Scienze Naturali e del Museo Civico di Storia Naturale di Milano. Vol.141. Fasc.2. P.291-301.

Spiegelaere de W., Bosmans R. 2009. Spider fauna in a grass dune remnant at the Black Sea cost (Romania), presenting seven new species for the romanian fauna including the first description of the female Harpactea alexandrae Lazarov, 2006 (Dysderidae) // Analele Șțiintifice ale Universitatii "Al. I. Cuza" Iaşi. Sect. Biologie animala. T.55. P.7-16.

Responsible editor D.V. Logunov 